
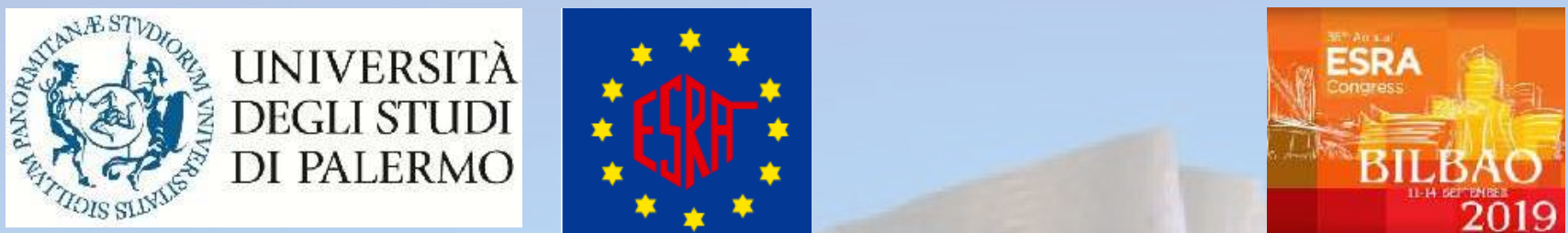

\title{
THE ERECTOR SPINAE PLANE BLOCK AS A ANALGESIC REGIONAL TECHNIOUE IN ACUTE POST-SURGICAL PAIN CONTROL IN LUMBAR SURGERY. PRELIMINARY FINDINGS OF A RANDOMIZED TRIAL.
}

L. La Brocca1, R. Calia1', M. Ventimiglia1, C. Marino1, G. Favaro1, T. Catania Cucchiara1, R. Catalfio¹, B. Ravazzolo3, D. Canzio 2.

1Section of Anaesthesia- Analgesia- Emergency- Intensive Care. Palermo University Medical Center- Italy. A. Giarratano, Full Professor 2Department of Surgical- Oncological and Oral Science Di.Chir.On.S., Palermo- Via del Vespro 129. 90127, Italy.

3Department of Health Promotion Sciences, Maternal and Child Health, Internal Medicine and Specialist Excellence "G.D'Alessandro". Via del Vespro 129. 90127, Italy.

\section{BACKGROUND AND AIM:}

Severe post-operative pain is often referred by patients undergoing posterior spine surgery. Inadequate pain control affects morbidity and hospitalization length of stay.

Post-operative pain in adults is commonly managed with oral and intravenous opioids with important side effect, and invasive regional techniques such as epidural blockade. Recent data has shown that the novel erector spinae plane (ESP) block, can be employed as a simple, effective and safe alternative regional analgesic technique for acute post-surgical, post-traumatic and chronic neuropathic lumbar pain in adults.

The aim of the present study was to investigate the capacity of ESP block to provide successful post-operative pain management following lumbar surgery, and to assess pain scores in patients undergoing spinal surgery.

\section{METHODS:}

29 patients undergoing open lumbar decompression surgery were randomized in two groups. ESP group $(n=12)$ received ESP block with 0,5\% levobubivacaine 20 $\mathrm{ml}$, in the control group ( $n=17$ ) no intervention was performed and postoperative analgesia was achieved with IV morphine plus ketorolac. The primary outcome was represented by postoperative numerical rate scale (NRS) score at various time periods during the first 24 hours following surgery. The secondary outcomes included opioid consumption, rescue analgesia and opioid related side effects. The study protocol was approved by the local review board.

\section{RESULTS:}

Compared with control group, the NRS score of ESP block patients did not show any statistically significant differences in the measured time periods. Twenty-fourhour opioid consumption in group control was significantly higher compared to group ESP ( $30 \pm 2.6 \mathrm{mg}$ and $10 \pm 2.08 \mathrm{mg}, \mathrm{p}<0.001$, respectively).

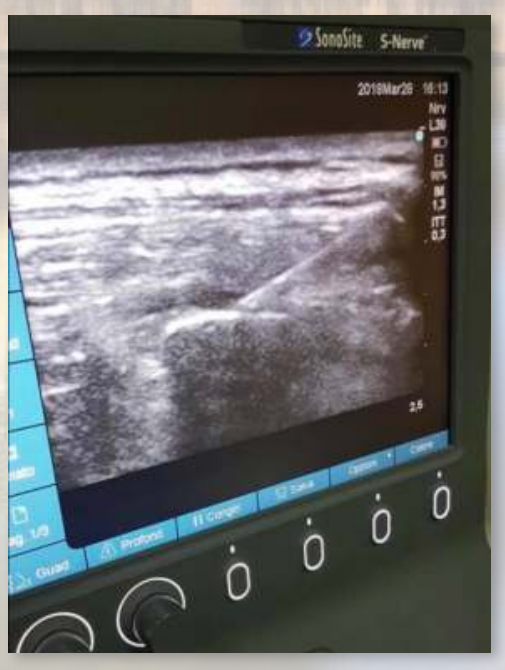

\section{CONCLUSIONS:}

Our preliminary findings suggest that ESP block is not inferior compared to opioid in the management of post-operative acute pain, has reduced opioid consumption and may play a key role in the prevention of post-surgical chronic pain.

\footnotetext{
References:

Hironobu U, Mayumi I, Tomoaki T, Hiroshi O. Efficacy of the Erector Spinae Plane Block for Lumbar Spinal Surgery. A Retrospective Study Forero M, Adhikary SD, Lopez H, Tsui C, Chin KJ. The Erector Spinae Plane Block: A Novel Analgesic Technique in Thoracic Neuropathic Pain. Ivanusic J, Konishi Y, Barrington MJ. A cadaveric study investi- gating the mechanism of action of erector spinae blockade. Reg Anesth Pain Med. 2018;43:567571. 